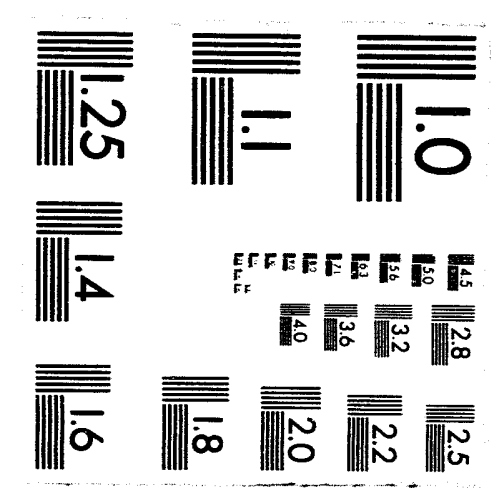



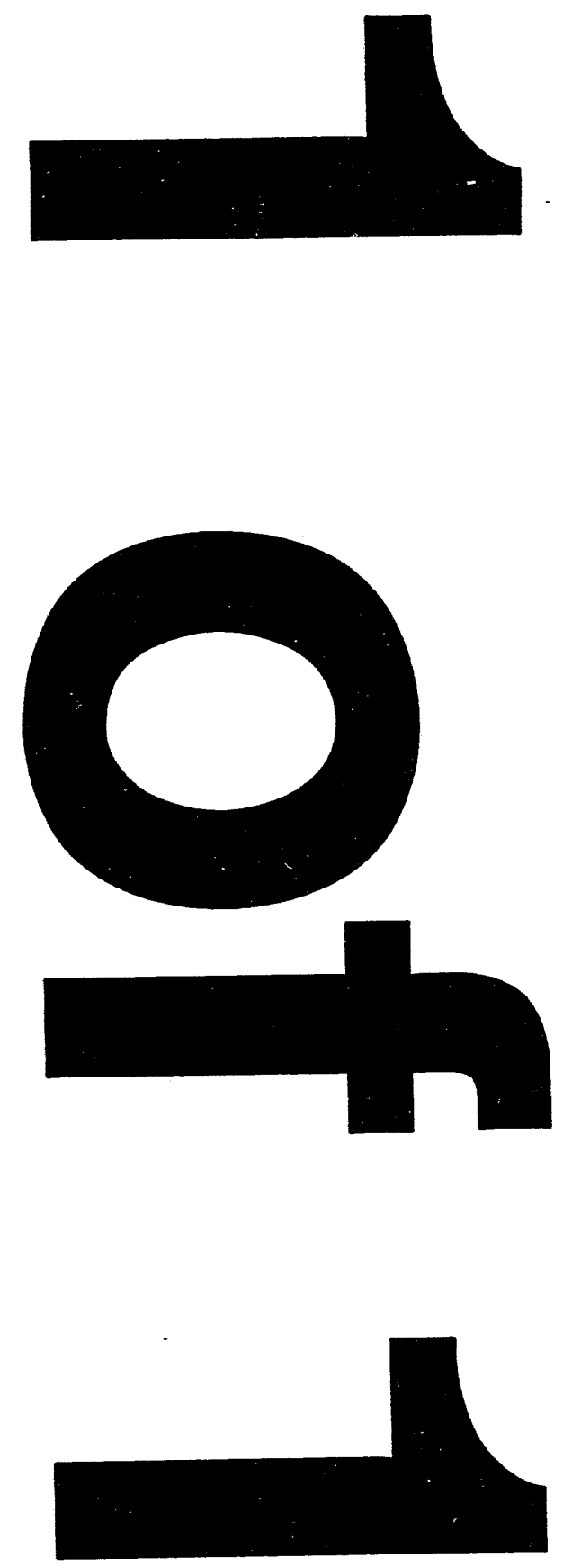


\section{$\operatorname{Con} f(310212 \ldots-1$}

- LA-UR- 93-3546

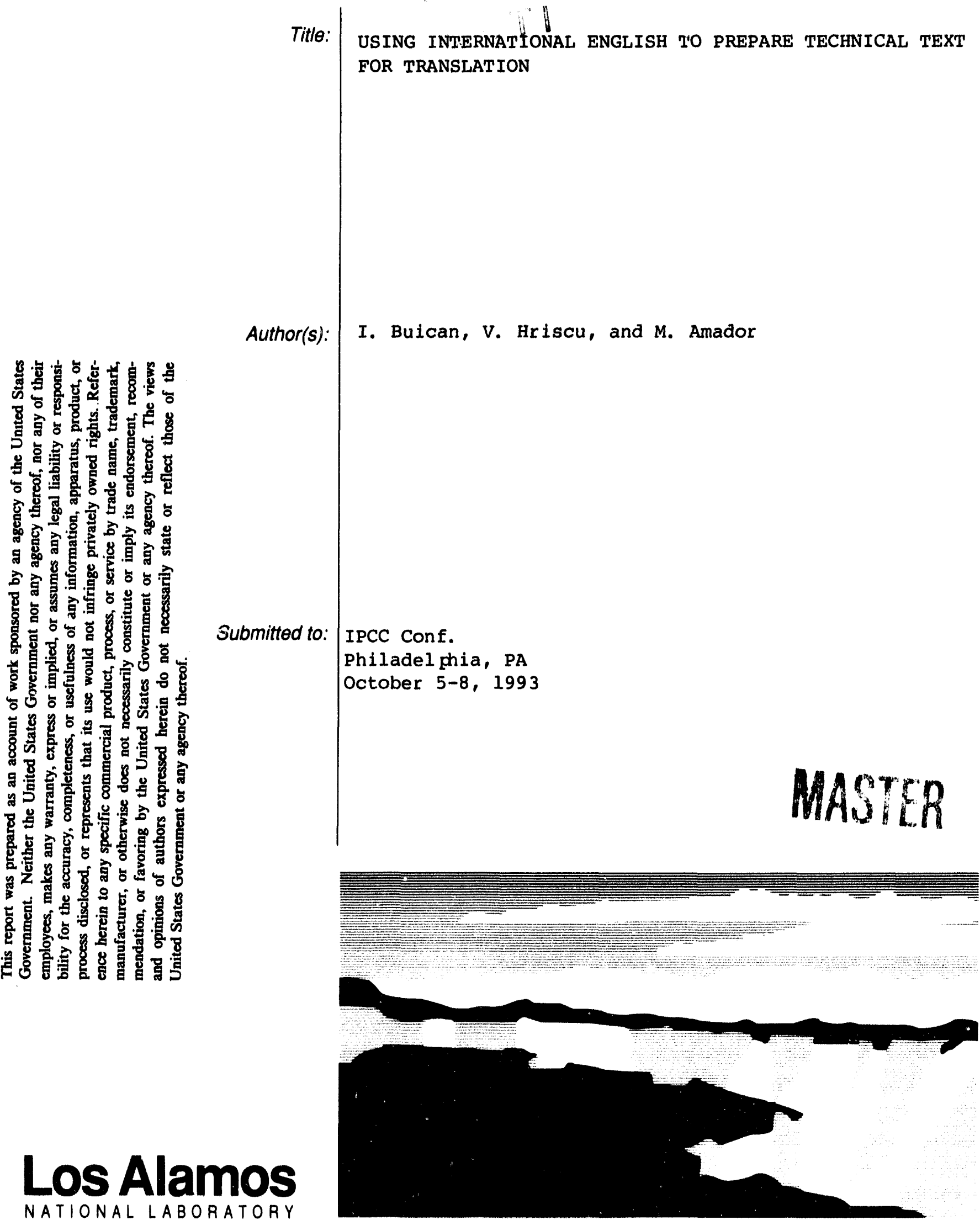

NATIONAL LABORATORY

Los Alamos National Laboratory, an affirmative action/equal opportunity empldyer, is operated by the University of California for the U.S. Department of Energy under contract W-7405-ENG-36. By acceptance of this article, the publisher recognizes that the U.S. Government retains a nonexciusive, royalty-free license to publish or reproduce the published form of this contribution, or to allow others to do so, for U.S. Government purposes. The Los Alamos National Laboratory requests that the publisher identify this article as work performed under the auspices of the U.S. Department of Energy. 


\title{
Using International English to Prepare Technical Text for Translation
}

\author{
by \\ Ileana Buican, Vivi Hriscu, and Mable Amador \\ Los Alamos National Laboratory
}

Communication and translation are facilitated when authors write in uncomplicated prose. Straightforward prose is easy to understand and translate; complicated, convoluted sentences and long, unpronounceable words make comprehension taxing and result in unintentional, yet often unavoidable, mistakes.

Having recognized the need for documents in English that are easy to read and understand, the International Communication Committee at Los Alamos National Laboratory has been working, for the past three years, to develop an expertise in and a methodology for writing documents in International English, that is, English for those whose native language is not English. So far, we have applied these guidelines to documents intended for presentation in English to an international audience of technical experts. We have also used them to improve the readability of English text written for a culturally diverse group of people at Los Alamos. We believe that the guidelines apply equally well to the preparation of technical text for translation because, usually, translators are native speakers of the target language, not of the source language. Considering that professional translators are primarily language experts, not technical experts, their difficilty in understanding original text written without an awareness of the needs for subsequent translation can have the undesirable but not surprising consequence of an inadequate rendering of the original concepts in another language. An easy and quick solution to 
the problem of the sentence given in our abstract would have been to change the word order of the original sentence (Viewgraph 1)

For warm springs, the enthalpy and silica content after mixing may be considered to be conservative estimates

to

For warm springs, the silica content and enthalpy after mixing may be considered to be conservative estimates.

Although the translators would still have been unfamiliar with the meaning of "enthalpy," they would not have changed, in the translated version, "the enthalpy and silica content" into "the content of enthalpy and silica." Nonnative-English-speaking scientists and engineers, will often be able to "fill in" when information is not crystal-clear; but in many cases translators will not, and we must bear that in mind when we prepare technical text for translation. Indeed, the only relevant standard by which to judge written text is the ease with which it conveys its full intended sense to its readers, be they technical experts or translators, who, as intermediaries, must communicate those concepts accurately to technical experts.

International English offers a set of practical guidelines specifically designed and formulated to address areas of written or spoken English that pose particular problems for nonnative speakers of English. We continue to work on our guidelines by adding new areas of interest to nonnative speakers and by refining those areas we have already covered. A quick look at our guidelines will show that the guidelines overlap in many respects with "general rules for good writing." However, our aim is to 
isolate those areas of language particularly important for nonnative speakers of English and to focus on the sources of confusion that are most clearly related to cultural and linguistic differences.

The workshop will consist of three parts: first, we will present the guidelines that seem most pertinent to preparing English text for translation. Second, we will apply the guidelines to a technical document intended for translation into Russian. Finally, you will have the opportunity to apply the guidelines discussed to several short documents intended for translation into Russian or Spanish. We will also show you a passage that has been translated from French into English so that you can better understand how a translation reads when the original is not clear.

A. We recommend the following specific guidelines for VOCABULARY:

\section{Avoid idioms.}

Idioms are phrases that cannot be understood from the meanings of the individual words that are included in them. It is not at all unusual to come across an example of technical writing that uses idiomatic expressions understood in different ways even by native speakers themselves. A sentence such as

(Viewgraph 4)

To all intents and purposes, numerical modeling in science and engineering involves a small number of basic steps...

raised a few eyebrows when we tested it on a number of scientists. To all intents and purposes is, of course, a filler phrase, one of those phrases 
we use when we cannot think clearly, but it is also an idiom. What does it mean? It can mean "apparently", "as far as we can tell," "in fact," or "in most ways." Which one of these meanings is the reader or listener supposed to choose? Our point is that the use of idiomatic expressions is conducive to vagueness of meaning, a feature that we should try and reduce in any writing and particularly in technical writing meant for people whose first language is not English. Let us look at some more examples:

(Viewgraph 5)

The conventional-boiler cost information was obtained from ruleof-thumb estimates supplied by ...

Most of the native English speakers we asked to define rule of thumb in this context told us that the phrase meant "standard" or "norm." In fact, rule of thumb implies a result obtained by guesswork based on experience. Empirical may have been a more accurate substitute. This sentence comes from a paper that was subsequently translated into Spanish. It is interesting to notice that the Spanish translator left out the troublesome word.

Some verbs followed by obligatory prepositions are idiomatic. Because they cannot be understood from the meanings of the individual constituent parts, International English needs to replace them with simple verbs.

(Viewgraph 6)

to put up with $=$ to tolerate

to have to do with $=$ to involve 
to turn down $=1$. to diminish the speed, volume, intensity, or flow of

2. to reject or refuse

\begin{abstract}
2. Do not use the same technical term to name two or more different concepts. Do not use more than one technical term to name the same concept.
\end{abstract}

Technical words used in two or more different senses within the same document are another source of confusion for an international audience.

(Viewgraph 7)

In the pacemaker industry, for example, the entire lead, that is, the wire that connects the pacemaker to the heart, is referred to by many specialists as the electrode (A). However, the same term, electrode, is also applied to the end of the lead that conducts electrical impulses to cardiac tissue (B). Moreover, the word connector is often used for both a part of the pacemaker $(C)$ and a part of the lead (D). As a result, a sentence such as

(Viewgraph 8)

Lubricate the entire outer portion of the connector is difficult to interpret. What must we lubricate? Is it $\mathbf{C}$ or $\mathbf{D}$ ?

Because it is confusing to have the same term define two different parts of a piece of equipment, the translation coordinator for these pacemaker documents rewrote parts of the original by observing International English guidelines. She called the whole wire lead and only its stimulating end electrode. Then, she left the word connector 
for the lead and used the term lead receptacle for the pacemaker because, as she found out, that was precisely the function of that part of the hardware. Only afterwards could she proceed with the translation. Indeed, considerable time and money could have been saved had the original text been written in International English.

3. Avoid using as part of your general vocabulary a term that has both a technical and a general meaning. Use a synonym for the general term.

It may be confusing, at least at first sight, to read (Viewgraph 9)

Current physical characterization and testing of hightemperature superconducting (HTS) devices include routine measurements of resistance versus critical current density.

The first current is a general word, an adjective that means present. The second current, however, is a technical term, a noun meaning electrical current. Because of possible lack of clarity, we recommend that writers of International English replace the general word with a synonym.

4. Avoid useless jargon. ( a pretentious or unnecessarily obscure and esoteric terminology; language vague in meaning and full of circumlocutions and long, high-sounding words)

Each profession has, undoubtedly, its own jargon, its set of technical terms that may not necessarily be understood by people outside the respective profession. Because scientific and technical discoveries are born 
with such speed, technical people permanently coin new terms to reflect new ideas. Therefore, the use of technical jargon is unavoidable in technical documents. For example, in a document coming from the computer industry, we may encounter a sentence such as

(Viewgraph 10)

A bug in the program delayed our research considerably.

A bug in a computer program is, of course, an error that makes the program run incorrectly. Nobody in the computer industry will refer to that type of error by a term other than bug.

Although such a word may be preserved in a document written in International English because it truly reflects what is happening in a certain profession, we recommend that its meaning be defined in a Glossary of Terms. What we need to guard against, however, is useless jargon, which appears in technical documents often. Let us consider the following example:

(Viewgraph 11)

Due to incompetence, the machine was rendered inoperative

could easily have been rewritten as

The machine broke because the users were incompetent.

And one more example:

(Viewgraph 12)

Successful completion of this course will be based on $100 \%$ proficiency on a performance test. 
The sentence would have been so much more straightforward as:

To pass this course, the participants must get a perfect score on the final test.

To sum up, there is a category of useful jargon that we do not need to avoid, but we need to define for our international audience; and there is also useless jargon that International English should avoid.

(Show cartoon-Viewgraph 13)

When undefined and used excessively, acronyms, abbreviations, and initials make us laugh because they seem absurd or irritate us because they mystify us. Therefore, our guideline is

\section{Avoid acronyms, abbreviations, and initials.}

(Viewgraph 14)

We also plan to help BCTP explore the use of video in the TOC to reinforce $\mathrm{O} / \mathrm{C}$ observation of events.

Although frequently used as shorthand, acronyms, abbreviations, and initials can impede communication for native and foreign English-speaking people alike. For International English, our advic: is to avoid such shorthand as much as possible and to define it in a Glossary of Terms if usage is mandatory.

All acronyms are pronounceable, as words are, but there are some acronyms that have become words through frequent use. For example, it is 
probable that few of the people asked what radar stands for will remember that the word is, in fact, an acronym for

(Viewgraph 15)

radio detecting and ranging.

The same holds true for scuba and laser. Acronyms such as these three, which are used in all languages as words, will not have to be defined, but their case is special. Most people will be surprised to hear that those words are acronyms.

Initialism is a shorthand created by writing the initial letters of a phrase. The result is not pronounceable as a word.

The following sentence,

(Viewgraph 16)

Eight companies responded to an RFP, and their proposals are now under review,

should have been written as

Eight companies responded to a request for proposal (RFP), and their proposals are now under review.

\section{Avoid wrong diction.}

This is another requirement for all good writing. However, technical writing for an international audience should pay special attention to this guideline. If native speakers may deduce the meaning of a sentence that contains diction errors from the context in which the sentence appears, nonnative speakers of English will likely be confused by such errors.

(Viewgraph 17)

The group compresses a few editors and technical experts. 
(Viewgraph 18-cartoon)

In fact, the author simply meant to say

The groups consists of (comprises) a few editors and technical experts.

(Viewgraph 19)

The expected shelf life of silicone rubber is in excess of two years.

It should be stored in an unopened container, in a dry place, at less than $80^{\circ}$ Fahrenheit.

The expected shelf life of silicone rubber is over two years. This material should be left in its factory-sealed container, in a dry place, at less than $\mathbf{2 7}^{\circ} \mathrm{C}$.

\section{Avoid wordiness.}

One of the outstanding features of English is its relative lack of inflexions-this means that relationships among words in a sentence are indicated with only minimal changes in the appearance of words. However, such relationships have to be indicated somehow. That is why English, much more than other European languages, is prone to periphrases, which are roundabout ways of expressing ideas. It seems to us that this is at least one of the reasons for so much wordiness in poor-quality technical writing in English. Let us consider the following examples:

(Viewgraph 20) 
The opening used to introduce the mixture into the system may be small and there may be no other openings where air can escape in such cases, the original opening may need to be enlarged or another opening created to allow air to escape.

Problems:

1. wordiness (used to introduce)

2. sentence structure (compound/complex)

3. wrong diction (where)

4. punctuation (period after "escape")

5. passive voice ("may need to be enlarged" and "may need to be created")

If the opening for introducing the mixture into the system is too small for air to escape, enlarge it or create a second one.

Periphrases, circumlocutions, and expletive constructions-there is or there are-or phrases such as it is necessary to are all conducive to wordiness.

Finally, smothered verbs (verbs that stifle under the weight of a long string of words) create wordiness and ambiguity. Avoid them.

(Viewgraph 21 -cartoon)

B. Moving from vocabulary in general to the more specific topic of NOUNS, we need to stop and consider overmodified nouns, which constitute one of the most troublesome problems of technical writing for an international audience. Our guideline is

1. Avoid overmodified nouns. 
Lack of inflexions in English makes it difficult for the reader or listener to understand the relationships among a number of nouns and adjectives that modify one noun.

(Viewgraph 22)

This low viscosity silicone rubber compound flows easily, and penetrates well into small cracks.

This low-viscosity silicone-rubber compound flows easily and penetrates well into small cracks.

Because of its low viscosity, this silicone-rubber compound flows easily and penetrates well into small cracks.

\section{Use articles correctly.}

The meaning of a sentence can radically be affected by the use or lack of use of definite and indefinite articles. Accurate usage of such articles is indispensable if an international audience is expected to understand the intended meaning correctly.

(Viewgraph 23)

Little change in the measured values indicates that the lead is well anchored in the ventricle.

The implication is that any change in values should be negligible and there may actually be no change.

If we introduce the indefinite article and say 
A little change in the measured values indicates that the lead is well anchored in the ventricle

the meaning of the sentence will be different, in fact, essentially opposite. The sentence implies that there should be some change if the lead is well anchored.

C. Another specific area of vocabulary includes VERBS. Our first guideline refers to voice.

\section{Use the active voice whenever you can.}

Technical editors lament the abuses of the passive voice in technical documents. Of course, excessive use of the passive is related to the tradition of scientific objectivity. Scientists are taught that technical papers need to be factual, impersonal, objective. What better way, the argument goes, than to express your statements in the passive.

This being said, we need to add that the passive voice has, indeed, its legitimate uses, as in the following example:

(Viewgraph 24)

Equal parts of the two chemicals were diluted in water as the experiment proceeded.

What happens to the two chemicals is the focus of the sentence and thus deserves emphasis. 
Most often though, technical experts fill their documents with passive constructions because of their unwillingness to express information directly:

(Viewgraph 25)

If it does not fully gel but remains watery or slightly liquid the remaining product should be discarded and replace with new silicone rubber components.

Rewrite:

Test the remaining silicone rubber to see if it gels fully. If it stays watery or slightly liquid, discard it.

2. Use correct tenses to express the relation of one action to another in time.

(Viewgraph 26)

The reaction occurred much faster in the second and third experiments, leading us to conclude that we did not use enough heat in the first one.

The reaction occurred much faster in the second and third experiments, leading us to conclude that we had not used enough heat in the first one.

Good writing style in English calls for variation of sentence length. Controlled or simplified versions of English advocate the use of only short sentences in an attempt to make the information clear to an audience with a 
low level of 'iteracy. In International English, we use both long and short sentences, but not without a word of caution: long sentences should not be compound/complex (namely, sentences that contain two or more independent clauses and one or more dependent clauses). Our rule therefore is

1. Vary sentence structure and length, but make sure that long sentences are not compound/complex.

(Viewgraph 27)

All these gases are inert and do not present any hazards, but if they are used in small, unventilated areas that are accessible to personnel, the gases could be asphyxiant hazards.

All these gases are inert and thus present no hazards. However, they can cause asphyxiation if they are used in small, unventilated areas. We can make long sentences readable in different ways as well. (Viewgraphs 28 and 29)

2. Use correct punctuation and lists to make a long sentence or paragraph readable.

(Viewgraph 28)

The LINAC system shall consist of a collection of individual modules that are incorporated into a horizontal "rack." The rack or "coffin" would support and enclose all the modules that make up the system with the exception of the "external modules" which include the Control Module, the Local Warning Module and the Coolant Heat 
Exchanger Module, all separate from the coffin. The coffin modules shall consist of the remainder of the system including the modulator and $\mathrm{rf}$ and $\mathrm{x}$-ray systems and a module called the Interface and Power Module that shall incorporate all the power distribution and control and monitor functions. The system shall be deliverable in a standard $3 / 4$ ton pickup truck. This includes water and gas reservoirs, cables, the external modules, the modules in the system rack and its housing and shock mitigation system.

\section{What's wrong:}

- No attempt at listing items that belong in the same category;

- Use of more than one term to name the same concept (rack and coffin);

- Undefined acronym;

- Lack of organizational skills in presenting ideas;

- Careless punctuation;

- Redundancy; and

- Generally poor writing.

What can be done to improve the paragraph:

- Give the passage structure by organizing ideas in an acceptable order;

- Use a list and punctuate it correctly;

- Eliminate redundancy;

- Define acronym.

(Viewgraph 29-Rewrite) 
The linear accelerator (LINAC) system shall consist of

- several modules incorporated into a covered, horizontal rack that resembles a coffin. The rack modules shall include (1) the modulator, (2) the radio-frequency ( $r f$ ) system, (3) the $x$-ray generator, and (4) the interface and power module whose function is to distribute, control, and monitor power;

- three external modules: (1) the control module, (2) the local warning module, and (3) the temperature-conditioning module;

- water and gas reservoirs;

- cables,

- housing, and a

- shock-mitigation system.

The entire LINAC system shall be deliverable in a 3/4-ton (convert to tonnes) pickup truck.

Native speakers of English often leave out words from written or spoken sentences. Although such practice does not always result in obliteration of meaning, it can lead to ambiguity because the sentence is left grammatically incomplete. The technical term for this situation is ellipsis defined as "the omission of a word or words necessary for the complete syntactical construction of a sentence but not necessary for understanding it" (The American Heritage Dictionary). If other native speakers can deduce the meaning of sentences that contain ellipses, nonnative speakers may have a hard time doing the same. Therefore, the next guideline is

\section{Avoid ellipses.}


(Viewgraph 30)

The cylinder is shown dashed in Figure 4.

The cylinder is shown by a dashed line in Figure 4.

(Viewgraph 31)

The pot life and gel time will be longer if the mix rutio of twenty to one is increased and reduced if the ratio is decreased.

The pot life and gel time will be longer if the mix ratio of twenty to one is increased; they will be shorter if the ratio is decreased.

(Viewgraph 32)

Another potential problem is trapping air in a system that is tightly sealed.

Another potential problem is the trapping of air in a system that is tightly sealed.

Another potential problem is that air may get trapped in a tightly sealed system.

Another potential problem is how to trap air in a system that is tightly sealed. (This version may very well have been the result of the original ambiguity.) 
Because of the loss of inflexions in English, word order tends to be relatively fixed. Because of minimal changes in the appearance of words, relationships among words may prove to be ambiguous. A fixed word order serves, therefore, as a marker for proper relationships among words; it helps clarify meaning. Therefore the next guideline is

4. Use correct word order in English. When more than one correct order is possible, use the one that will lead to the least ambiguity.

(Viewgraph 33)

A fire in any zone signals the fire control panel, which then sends the concentrator a signal.

A fire in any zone signals the fire control panel, which then sends a signal to the concentrator.

(Viewgraph 34)

Everyone was glad when the computer broke for more reasons than one.

When the computer broke, everyone was glad for more reasons than one.

\section{(Viewgraph 35)}

He analyzed the data in the afternoon for two hours.

He analyzed the data for two hours in the afternoon. (Duration precedes a particular moment in time.) 
The last guideline we shall examine is

Use the International System of Units for numerical notations. (Viewgraph 19)

The expected shelf life of silicone rubber is in excess of two years. It should be stored in an unopened container, in a dry place, at less than $80^{\circ}$ Fahrenheit.

The expected shelf life of silicone rubber is over two years. It should be stored in a sealed container, in a dry place, at less than $27^{\circ} \mathrm{C}$. 


\section{ORIGINAL}

\section{LINAC SPECIFICATION}

\section{Background:}

The x-ray generator may be needed to examine objects in remote locations away from roads or facilities under extreme environmental conditions and demanding time frames. The generator must be as reliable and simple to operate as it can possibly be made. If conditions permit, the generator would be transported to the site as a unit, already cabled up, checked out and ready to go. Special conditions may dictate, however, that the generator be taken apart, transported to the site, reassembled and operated with a very high degree of confidence. This requires that the components of the generator be small, light, and well protected from moisture and shock. 


\section{REWRITE}

\section{Specification for a Linear Accelerator (LINAC) System}

\section{Background:}

The x-ray generator may be used to examine objects in remote locations, away from roads or facilities, under extreme environmental conditions, and/or within short and intense periods of time. Ideally, the generator should be transported to the site as a unit, with all its cables connected, checked out, and ready for operation. Under special conditions, the generator will need to be disassembled, transported to the site, and then reassembled for operation. For these reasons, the generator must be highly reliable and easy to operate, and its components must be small, light, and well protected from moisture and shock. 
$1 !$
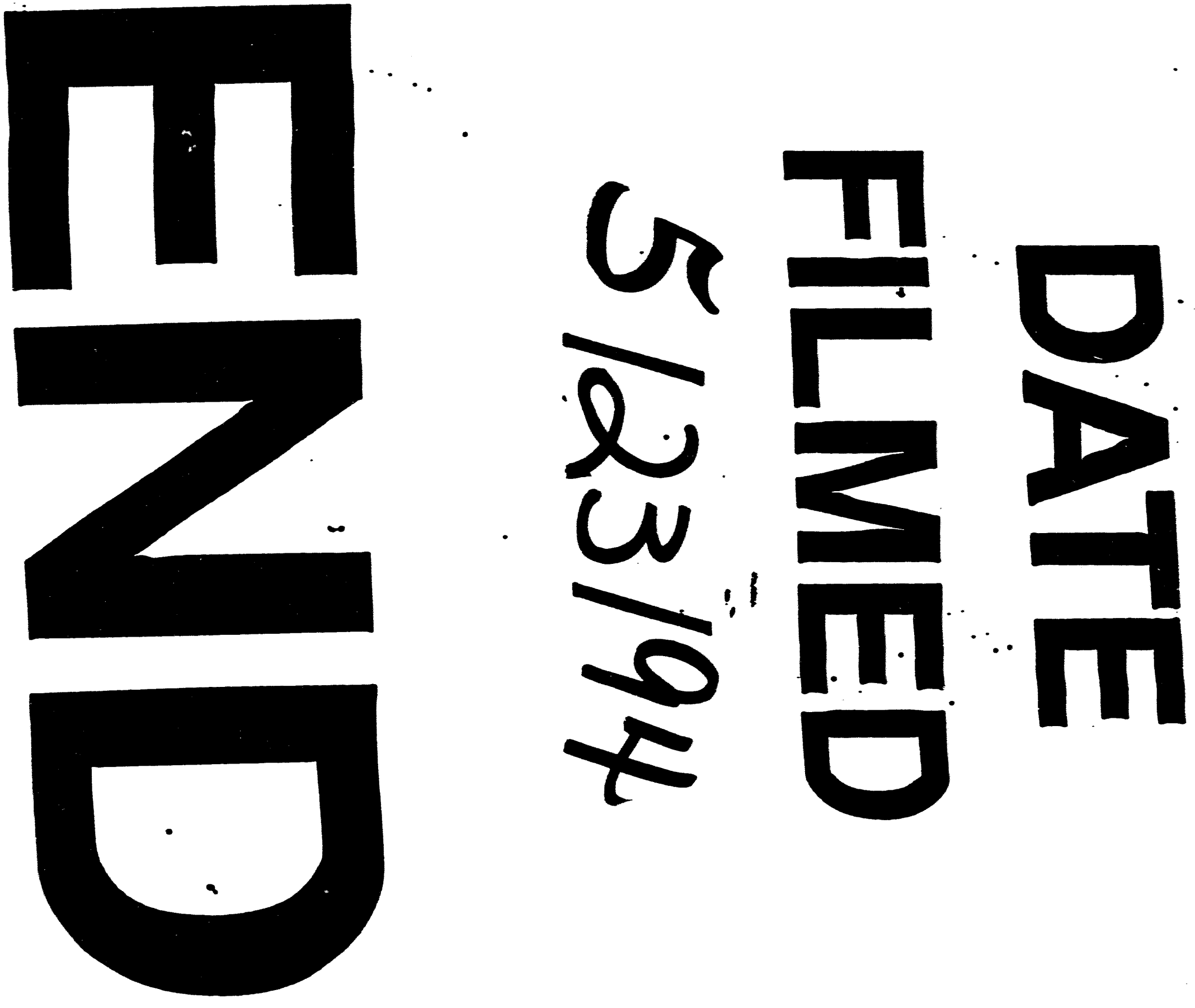


\section{$\overline{-}$

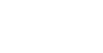

$$
-
$$

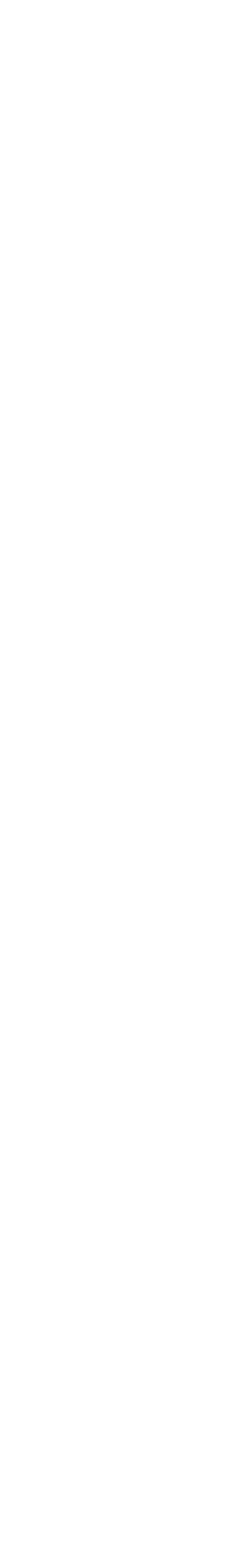

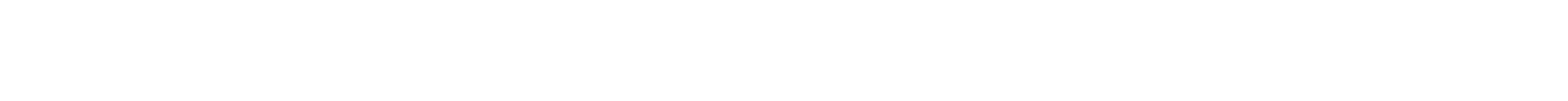

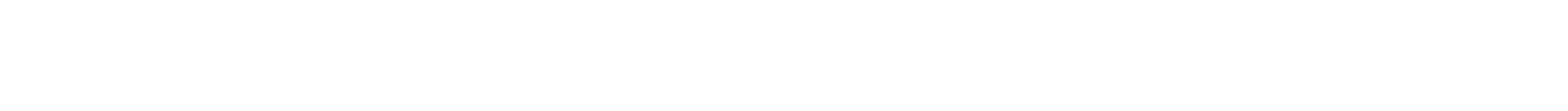

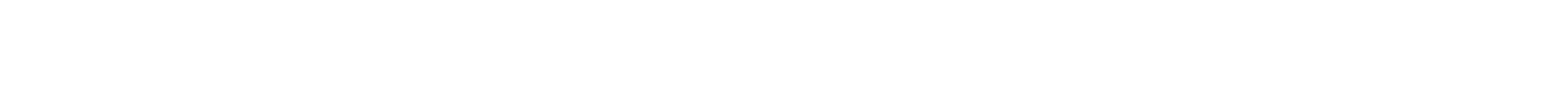

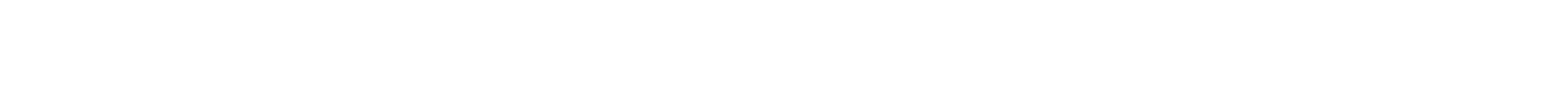

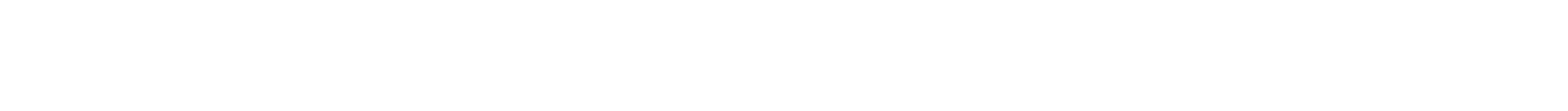

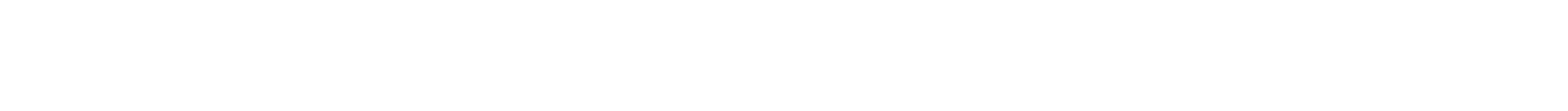

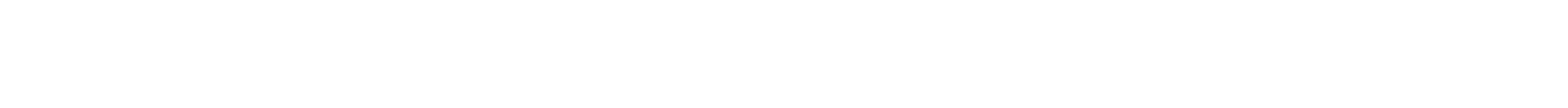

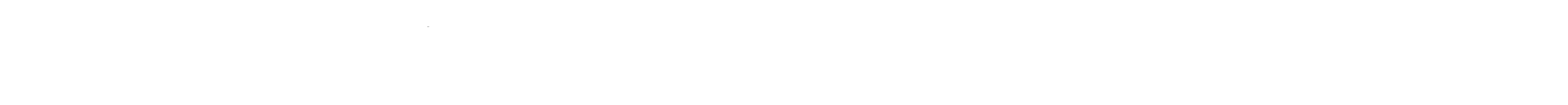

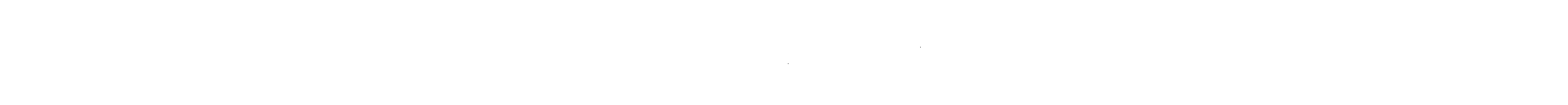

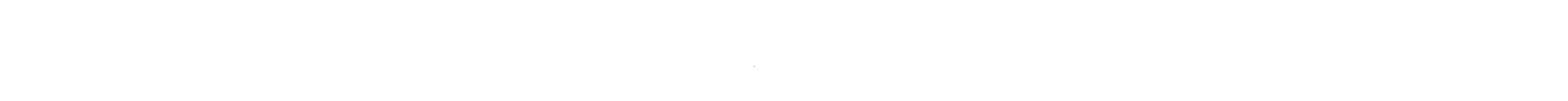

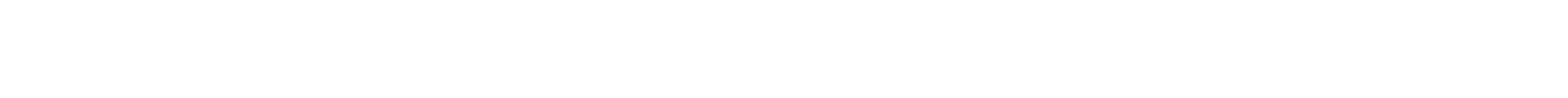

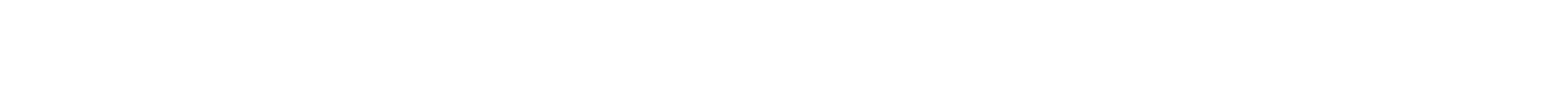

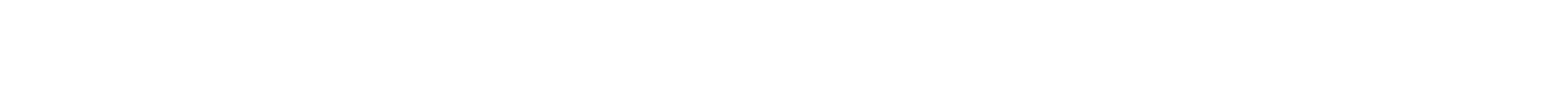

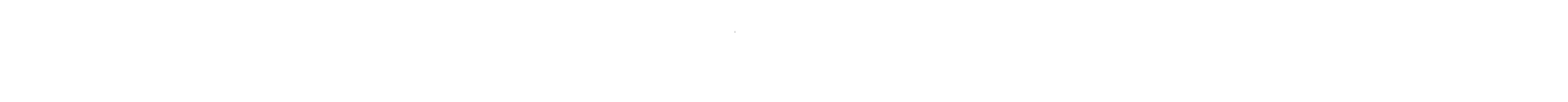

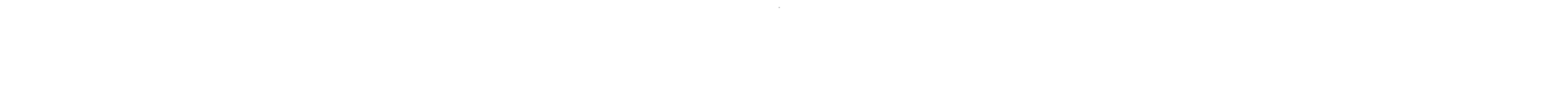

